\title{
Metalorganic Vapor Phase Epitaxy of III-Nitride Light-Emitting Diodes on Nanopatterned AGOG Sapphire Substrate by Abbreviated Growth Mode
}

\author{
Yik-Khoon Ee, Student Member, IEEE, Jeffrey M. Biser, Wanjun Cao, Helen M. Chan, \\ Richard P. Vinci, and Nelson Tansu, Member, IEEE
}

\begin{abstract}
Metalorganic vapor phase epitaxial (MOVPE) growth of GaN on nanopatterned AGOG sapphire substrates was performed, and characteristics of the light-emitting diode (LED) devices grown on patterned sapphire and planar substrates were compared. The nanopatterned sapphire substrates were fabricated by a novel process (AGOG) whereby aluminum nanomesas were epitaxially converted into crystalline $\mathrm{Al}_{2} \mathrm{O}_{3}$ via a two-stage annealing process. The GaN template grown on the nanopatterned sapphire substrate was done via an abbreviated growth mode, where a 15-nm thick, low-temperature GaN buffer layer was used, without the use of an etch-back and recovery process during the epitaxy. InGaN quantum wells ( $Q W s$ ) LEDs were grown on the GaN template on the nanopatterned sapphire, employing the abbreviated growth mode. The optimized InGaN QW LEDs grown on the patterned AGOG sapphire substrate exhibited a $24 \%$ improvement in output power as compared to LEDs on GaN templates grown using the conventional method. The increase in output power of the LEDs is attributed to improved internal quantum efficiency of the LEDs.
\end{abstract}

Index Terms-Dislocation density, InGaN quantum wells, lightemitting diodes, MOVPE growth, nanoheteroepitaxy, sapphire.

\section{INTRODUCTION}

$\mathbf{S}$ APPHIRE is most commonly used as the substrate for nitride light-emitting diodes (LEDs) in solid state lighting due to its physical robustness and high temperature stability. GaN native substrates are still at present very expensive and not viable for large-scale device production, in particular for addressing low-cost, solid-state lighting applications. Direct growth of high-temperature GaN on sapphire often results in poor film quality and severe epitaxy film cracking. This is due to the large lattice mismatch of $16 \%$ between wurtzite $\mathrm{GaN}$ and $c$-plane sapphire. To overcome this, conventional GaN growth on sapphire by metalorganic vapor phase epitaxy (MOVPE) employs low-temperature GaN buffer [1] or AlN buffer [2] layers, prior to the growth of high-temperature $\mathrm{GaN}$ layer. The essence of

Manuscript received December 29, 2008; revised February 11, 2009. First published May 26, 2009; current version published August 5, 2009. This work was supported in part by the National Science Foundation (NSF) under the Division of Electrical, Communications, and Cyber Systems (ECCS) Award 0701421, in part by the NSF under the Division of Materials Research (DMR) Award 0705299, and in part by Peter C. Rossin Assistant Professorship.

Y.-K. Ee and N. Tansu are with the Center for Optical Technologies, Department of Electrical and Computer Engineering, Lehigh University, Bethlehem, PA 18015 USA (e-mail: Ee@Lehigh.Edu; Tansu@Lehigh.Edu).

J. M. Biser, W. Cao, H. M. Chan, and R. P. Vinci are with the Center for Advanced Materials and Nanotechnology, Department of Materials Science and Engineering, Lehigh University, Bethlehem, PA 18015 USA.

Color versions of one or more of the figures in this paper are available online at http://ieeexplore.ieee.org.

Digital Object Identifier 10.1109/JSTQE.2009.2017208 the low-temperature, buffer-layer technique is to reduce the interfacial free energy between the epitaxial layer and the highly mismatched substrate by using a more compliant softer material [3]. To reduce the dislocation density of MOVPE GaN through the in situ method, hydrogen $\left(\mathrm{H}_{2}\right)$ etch back of the low-temperature $\mathrm{GaN}$ buffer layer and intentional delay of the nucleation island coalescence (recovery) is often adopted [4]. The etch-back and recovery technique typically adds 30 to 45 min to the MOVPE GaN growth time, thus adding significant cost to the epitaxy of nitride-based LEDs.

The threading dislocation density of the GaN template grown by the conventional approach is still high, in the mid $10^{8}-$ $10^{10} \mathrm{~cm}^{-2}$ range [5]. High density of threading dislocations has been linked to failure of lasers [6] and breakdown of $p-n$ junctions [7]. Hence, for high performance and reliable nitridebased LEDs and laser diodes, threading dislocation density has to be reduced. Several approaches have been implemented to reduce the threading dislocation density in GaN epitaxial layers, such as lateral epitaxial overgrowth (LEO) [8], pendeo epitaxy [9], and cantilever epitaxy [10]. These approaches have demonstrated reduced dislocation densities in the GaN layers in the range of $10^{6}-10^{7} \mathrm{~cm}^{-2}$, but the low-defect regions are only limited to 5-10 $\mu \mathrm{m}$ wide stripe ridges. Recently, Hersee et al. proposed a technique based on nanoheteroepitaxy (NHE) of $\mathrm{GaN}$ on nanopatterned silicon substrates [11], which leads to reduction in the dislocation density of the GaN film through the introduction of compliant substrate surface structures. Other research groups have also grown III-Nitride LEDs via conventional GaN growth on patterned sapphire substrate for the goal to increase the light extraction efficiency of LEDs [12]-[15]. However, the approach to enhance the light extraction efficiency requires relatively deep etch patterns in the range of $1-5 \mu \mathrm{m}$, as well as typical pattern size and center-to-center spacing of 3 and $4 \mu \mathrm{m}$, respectively [12]-[15].

In this paper, we present NHE of GaN template grown on nanopatterned AGOG $c$-plane sapphire substrate employing a novel "abbreviated growth mode" by MOVPE. We also compare the device characteristics of nitride LED devices grown on our current template with those grown on conventional GaN templates.

The advantages of using nanopatterned sapphire substrates are twofold: 1) increased internal quantum efficiency of nitride LEDs with improved reliability from the reduction in dislocation density and 2) ability to utilize the novel abbreviated growth mode for the epitaxy of $\mathrm{GaN}$ that avoids the need for 


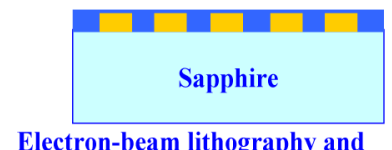

Electron-beam lithography and
Deposition of Aluminum $(\sim 100 \mathrm{~nm})$

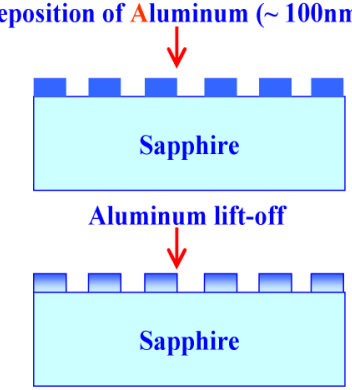

Growth of polycrystalline Oxide At $\sim 450^{\circ} \mathrm{C}$ in air

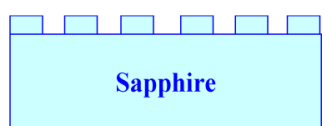

Grain growth in oxide at $\sim 1200^{\circ} \mathrm{C}$ in air Conversion to $\mathrm{Al}_{2} \mathrm{O}_{3}$ single crystal

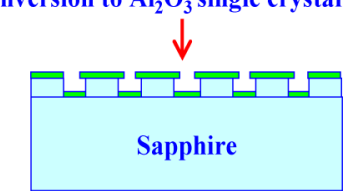

Pillar MOVPE Growth Mode of GaN on Patterned AGOG substrate

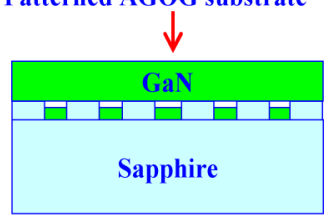

MOVPE Epitaxy of GaN layer on Patterned AGOG substrate

Fig. 1. Fabrication process of nanopatterned AGOG sapphire and MOVPE of $\mathrm{GaN}$ on nanopatterned AGOG sapphire substrate.

an etch-back and recovery technique. This, in turn, leads to a significant reduction in cost and time of the epitaxy. From our studies, we found that use of the abbreviated growth mode was important for optimized growth of $\mathrm{GaN}$ templates on nanopatterned sapphire, and improvements in LED output power were observed for the InGaN QW LEDs grown on nanopatterned sapphire substrates.

\section{NANOPATtERNING OF SAPPHIRE SUBSTRATE THROUGH NOVEL AGOG PROCESS}

In our approach, instead of patterning the sapphire substrate via an etching technique [12]-[15], we employed a novel AGOG process that enables creation of single crystal $\mathrm{Al}_{2} \mathrm{O}_{3}$ islands on the sapphire substrates. The "AGOG" is an acronym for the process of conversion $\mathrm{Al}$ into single crystal sapphire, by employing aluminum deposition, growth of oxide, and grain growth [16], [17]. It is the unique process of creating these nanostructures via oxidation of deposited aluminum (Al) islands to form $\mathrm{Al}_{2} \mathrm{O}_{3}$ and subsequently annealing to convert the islands to single crystal [16], [17]. In our current approach, by converting nanopatterned Al-metals deposited on sapphire substrate via AGOG process, nanopatterned AGOG sapphire substrate can be realized. The details of the nanopatterning of the sapphire via the AGOG process and MOVPE epitaxy of the GaN on the patterned AGOG substrate are presented in Fig. 1, with the following steps: 1) electron-beam lithography of metallic nanostructures on sapphire; 2) deposition of $100 \mathrm{~nm}$ Al-layer for lift off; 3) annealing treatment to grow a polycrystalline oxide; and 4) high-temperature grain growth.

In our experiments, the Al-metal lift off mask patterned by electron-beam lithography is composed of an array of hexagons approximately $200 \mathrm{~nm}$ wide with center-to-center spacing of $400 \mathrm{~nm}$. In our proof-of-concept experiment, the size of the patterned AGOG region was limited to $1 \mathrm{~mm} \times 1 \mathrm{~mm}$. A layer of $100 \mathrm{~nm} \mathrm{Al}$ was then deposited, followed by the lift off process. The patterned $\mathrm{Al}$ metal on $c$-plane sapphire underwent two heat treatments: 1) $450{ }^{\circ} \mathrm{C}$ oxidation anneal in air for $24 \mathrm{~h}$ [scanning

(a): After $450^{\circ} \mathrm{C}$ heat treatment (24 hours)

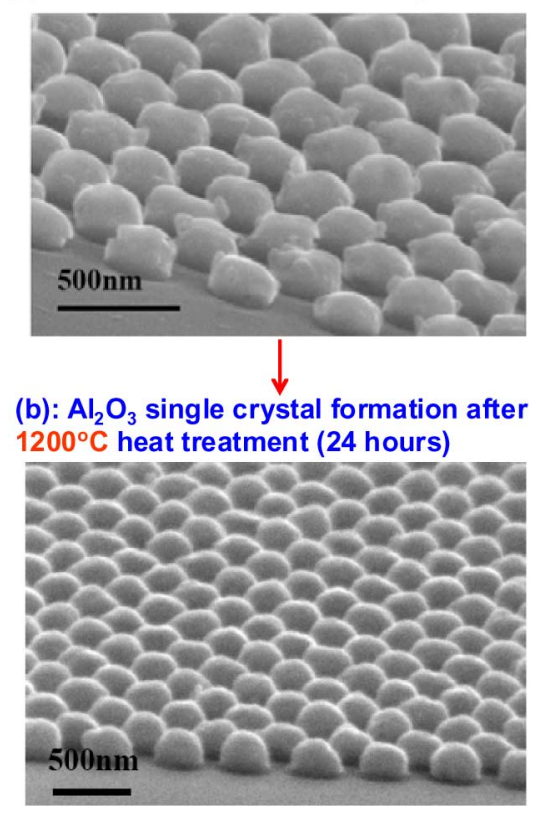

Fig. 2. (a) SEM of aluminum nanostructure array after oxidation at $450{ }^{\circ} \mathrm{C}$ and (b) epitaxial conversion to single crystal $\mathrm{Al}_{2} \mathrm{O}_{3}$ after $1200{ }^{\circ} \mathrm{C}$ anneal.
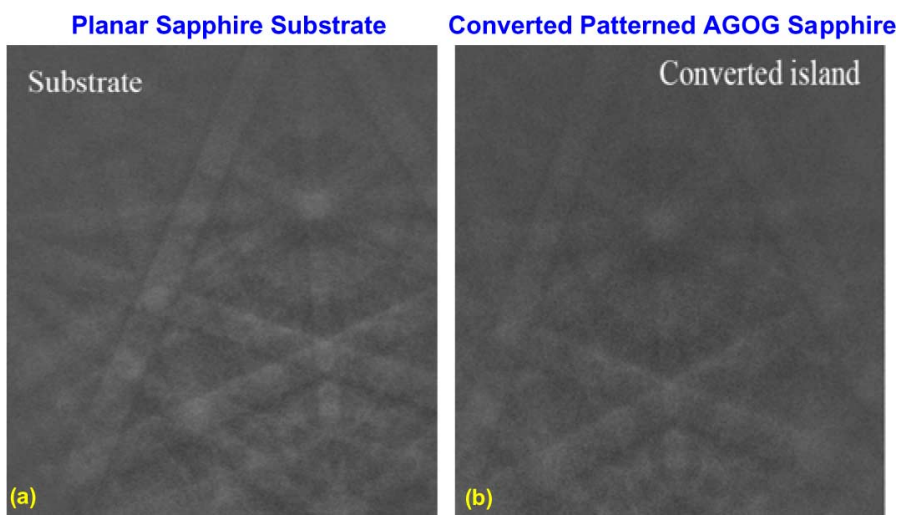

Fig. 3. Electron backscatter diffraction image of planar sapphire substrate and nanopatterned region after the AGOG process.

electron microscopy (SEM) image shown in Fig. 2(a)], followed by 2) $1200{ }^{\circ} \mathrm{C}$ in air for $24 \mathrm{~h}$ to induce grain growth of the underlying sapphire single crystal to consume the oxide layer [shown in Fig. 2(b)].

Electron backscatter diffraction (EBSD) was conducted on the planar sapphire [Fig. 3(a)] and nanopatterned AGOG sapphire [Fig. 3(b)] islands. Indexing of the patterns confirmed that the patterned AGOG nanostructures consisted of sapphire with the same orientation as the (0001) substrate [see Fig. 3(a) and (b)]. Further details on this finding have been reported elsewhere in [16]. The EBSD pattern of the AGOG nanopatterned region is not visible for cases in which the islands are not converted into single crystal. Hence, the EBSD pattern of the converted island in Fig. 3 was from the AGOG nanostructures and not the underlying sapphire substrate. The GaN layer was then grown on nanopatterned AGOG sapphire, using nanopillar growth mode. Ideally, the nanopillar growth mode enables the strain to distribute in three dimensions, which leads to a reduction in strain 
energy and stress-induced defect formation. The dislocations in the GaN grown on the patterned sapphire islands are terminated when the GaN film coalesces.

\section{MOVPE OF GaN Templates on Nanopatterned Sapphire Employing Abbreviated Growth Mode}

In the conventional $\mathrm{GaN}$ template growth, a $30-\mathrm{nm}$ thick, low-temperature $\left(535^{\circ} \mathrm{C}\right) \mathrm{GaN}$ buffer layer is grown on $c$-plane sapphire, followed by a $\mathrm{H}_{2}$ etch-back process, and a delayed recovery stage during high-temperature $\left(1080^{\circ} \mathrm{C}\right) \mathrm{GaN}$ growth. During the $\mathrm{H}_{2}$ etch-back process, the low-temperature buffer layer is broken down into micron-sized crystallites from which the high-temperature GaN can nucleate and grow. This step is critical for the successful growth of the high-temperature GaN. The AGOG nanopatterns on the sapphire substrate have much smaller dimensions at $200 \mathrm{~nm}$. Though the nanoisland size is not small enough to completely eliminate strain energy, it enables the strain to distribute in three dimensions, which leads to reduction in strain energy and stress-induced defect formation. Nonetheless, there is significant surface roughness introduced by the nanopatterned surface as compared to a standard planar sapphire substrate.

To investigate whether high-temperature $\mathrm{GaN}$ is able to nucleate on nanopatterned sapphire without the presence of a buffer layer, an experiment was conducted in which high-temperature GaN growth was carried out directly on a sapphire substrate with small AGOG nanopatterned regions surrounded by conventional planar surfaces. Fig. 4(a) and (b) show SEM images of $0.25-\mu \mathrm{m}$ thick, high-temperature $\mathrm{GaN}$ grown directly on this substrate. From Fig. 4(a), it was observed that the high-temperature GaN grows readily on the nanopatterned region of the sapphire. In contrast, the high-temperature GaN did not nucleate on the adjacent planar region. The scalloped appearance of the bottom edge of the nanopatterned sapphire in Fig. 4(a) is an artifact due to imperfect metal lift-off during processing. A higher magnification SEM micrograph of the high-temperature GaN grown on a nanopatterned region of the sapphire substrate is shown in Fig. 5. Together, these images confirm that the use of nanopatterned AGOG sapphire leads to improvement in the nucleation process of the $\mathrm{GaN}$ without the need for a low-temperature $\mathrm{GaN}$ buffer layer and etch-back and recovery process. However, closer examination of the high-temperature $\mathrm{GaN}$ grown in this way reveals that the surface has undesirable roughness on the $c$-plane surface.

As a compromise between surface quality and speed of growth, surface morphology studies were conducted using GaN grown on nanopatterned sapphire by an "abbreviated growth mode" technique. In this technique, a 15-nm thick, low-temperature $\mathrm{GaN}$ buffer is grown, followed by the growth of high-temperature $\mathrm{GaN}$ without the intermediate etch-back and recovery process. Fig. 6 shows SEM micrographs of the nanopatterned and planar sapphire after the growth of $15-\mathrm{nm}$ $\mathrm{GaN}$ buffer layer and $0.1 \mu \mathrm{m}$ of high-temperature GaN. The surface morphology of the two regions was very different. With a thin buffer layer - and with etch-back and recovery processes bypassed- $\mathrm{GaN}$ was preferentially grown on the nanopatterned
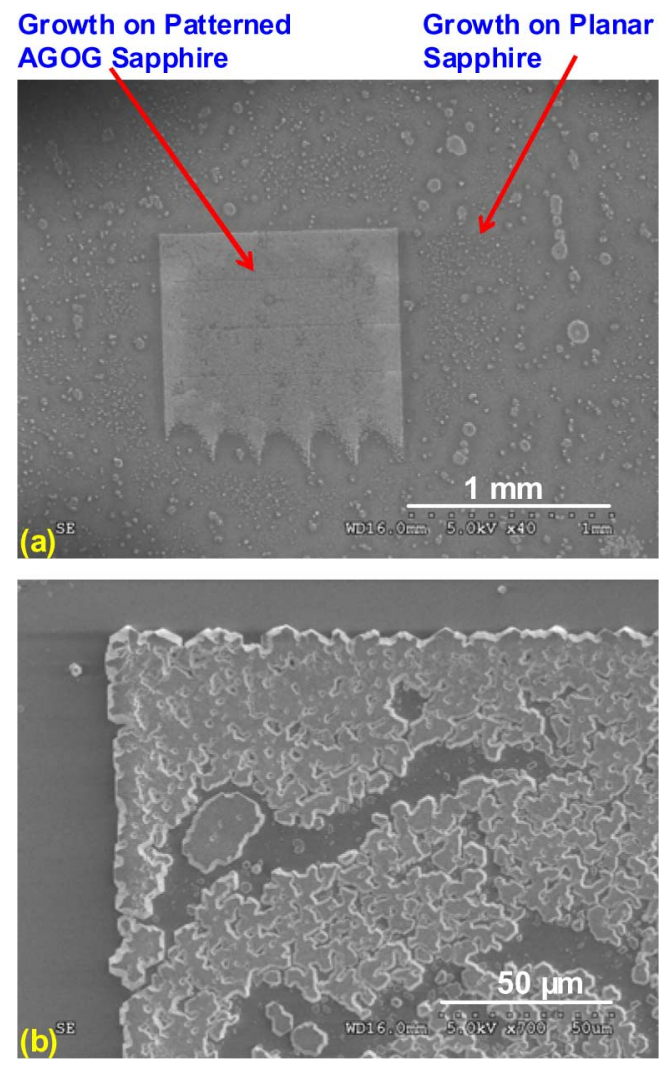

Fig. 4. SEM of (a) high-temperature $\mathrm{GaN}$ grown directly on the nanopatterned region as well as the surrounding planar region, (b) top left corner of nanopatterned region.

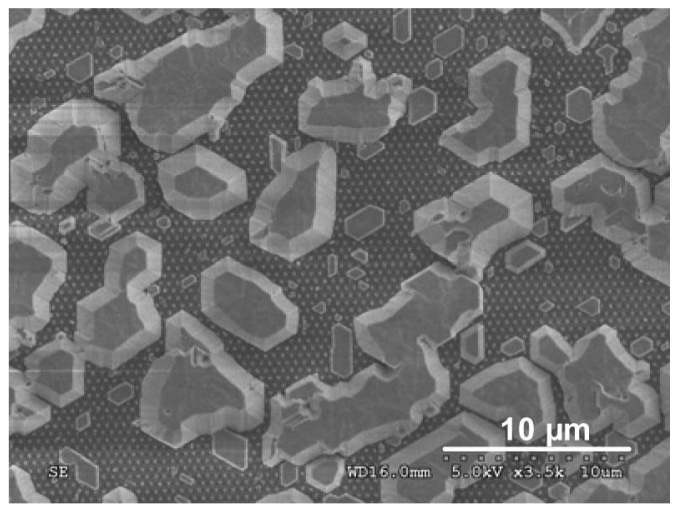

Fig. 5. SEM of 0.25- $\mu$ m-thick GaN grown directly on nanopatterned sapphire without a buffer layer.

sapphire region in a similar fashion to the case with no buffer layer. Despite the relatively thin GaN layer of $0.1 \mu \mathrm{m}$, coalescence of $\mathrm{GaN}$ on the patterned region was significantly advanced over that of the $0.25-\mu \mathrm{m}$-thick GaN grown directly on nanopatterned sapphire, resulting in relatively planar material early in the growth process.

To further investigate the surface morphology evolution during $\mathrm{GaN}$ growth, studies were also conducted on $0.25-\mu \mathrm{m}$ thick, high-temperature GaN grown on a similar nanopatterned sapphire substrate with a $15-\mathrm{nm} \mathrm{GaN}$ buffer layer. Fig. 7 shows a SEM micrograph of the GaN grown on a nanopatterned region of 


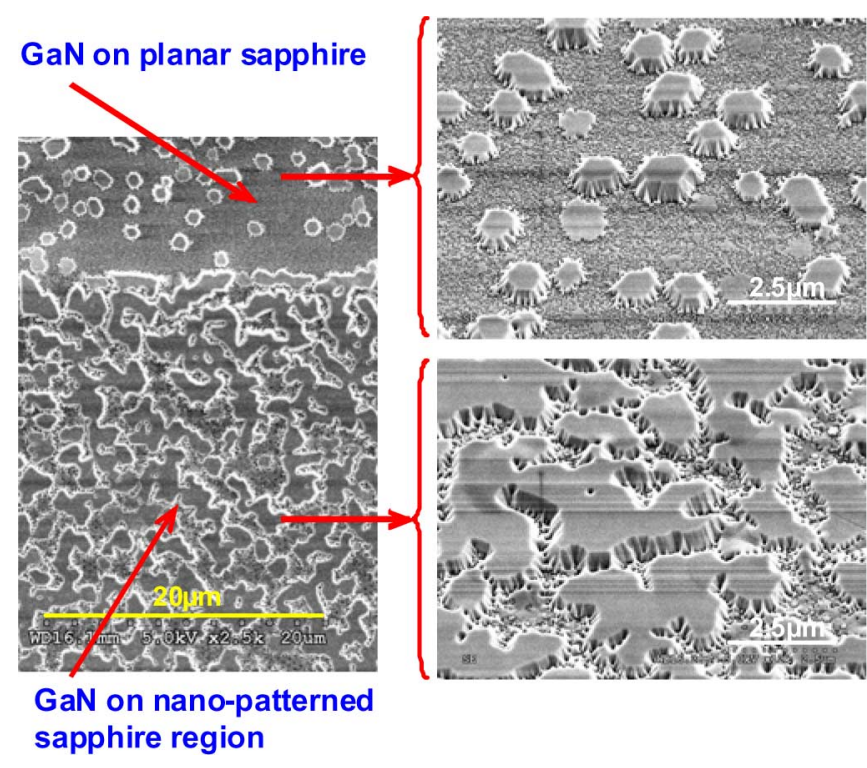

Fig. 6. SEM of $0.1-\mu \mathrm{m}$-thick GaN grown on nanopatterned sapphire and planar sapphire with a $15-\mathrm{nm}$ buffer layer.

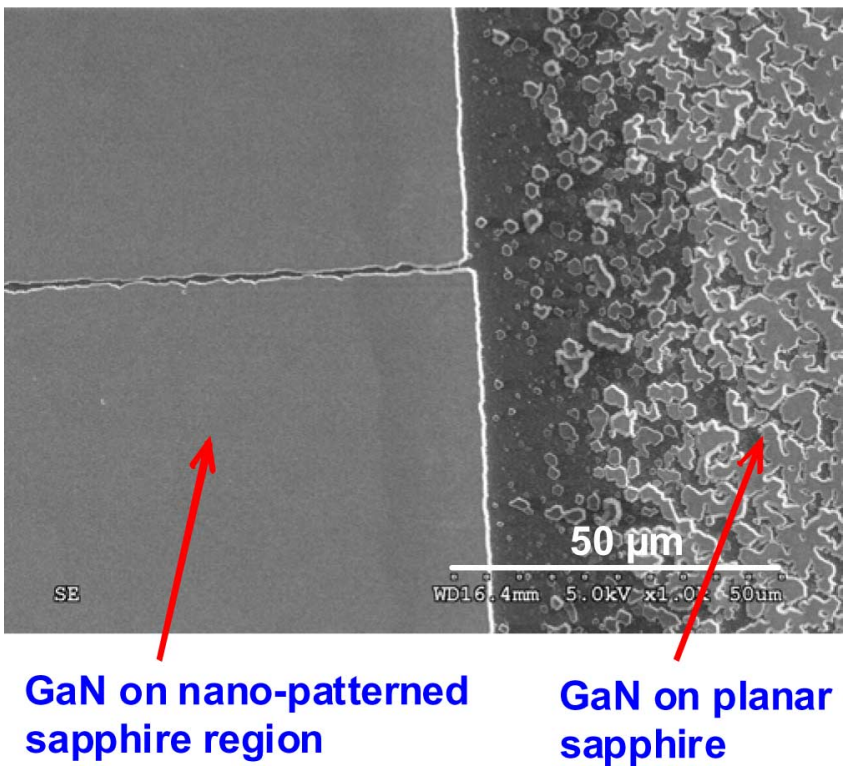

Fig. 7. SEM of $0.25-\mu$ m-thick GaN grown on nanopatterned sapphire and planar sapphire with a $15-\mathrm{nm}$ buffer layer.

the substrate, as well as that grown on the adjacent planar region. From Fig. 7, we observe that at $0.25 \mu \mathrm{m}$ the high-temperature $\mathrm{GaN}$ has completely coalesced forming a smooth film on the nanopatterned sapphire, but not on the planar region. The use of the abbreviated growth mode on nanopatterned sapphire is a significant advantage over the conventional approach since it reduces the epitaxy time, leading to cost reduction. Note that our abbreviated growth mode is different from the previously reported [18] GaN growth mode on etched patterned sapphire, which still require the use of conventional growth method utilizing the etch-back and recovery processes [18].

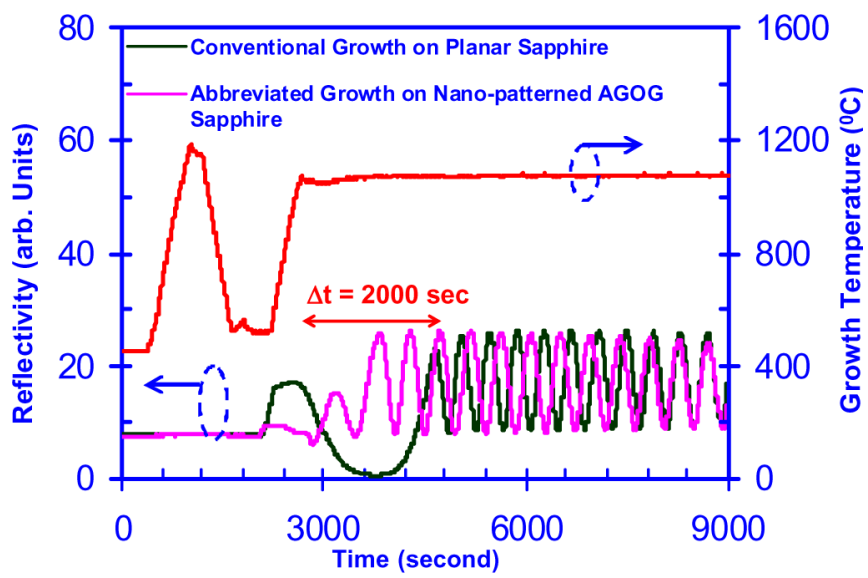

Fig. 8. Comparison of reflectivity during the growth of n-doped GaN template using the (a) conventional and (b) thinner buffer layer method without etch-back and recovery process. The temperature profile is shown for growth employing abbreviated mode.

\section{GROWTH OF InGaN QW LEDs}

As a first step toward fabrication of InGaN quantum well (QW) LEDs, the successful high-temperature $\mathrm{GaN}$ growth conducted on a thin buffer layer without the usual etch-back and recovery processes was extended to the growth of n-GaN templates on patterned and planar sapphire substrates. Conventional growth on planar sapphire was performed for comparison. During the growth of the high-temperature $\mathrm{GaN}$, the molar flow rate of trimethylgallium (TMGa) was $5.407 \mu \mathrm{mol} / \mathrm{min}$, and $\mathrm{NH}_{3}$ was used as group $\mathrm{V}$ source with a flow rate of $2800 \mathrm{sccm}$, corresponding to a V/III ratio of about 3700 . Fig. 8 shows a plot of reflectivity and growth temperature during epitaxial growth of the n-doped GaN templates. For the conventional growth, the etch-back and recovery stages are shown in the reflectivity curve. These growth steps were bypassed during the abbreviated growth for a savings of approximately $30 \mathrm{~min}$. The $\mathrm{GaN}$ templates grown using the conventional and abbreviated methods were both $2.8 \mu$ m thick.

The InGaN QW LED devices were grown on the three comparison $\mathrm{GaN}$ templates as follows: abbreviated growth mode on nanopatterned AGOG sapphire (sample 1), conventional growth mode on planar sapphire (sample 2), and abbreviated growth mode on planar sapphire (sample 3). The InGaN QW LED structures were grown on the comparison $\mathrm{GaN}$ templates at the same time, and the active region consisted of four-period $2.5-\mathrm{nm}$ $\mathrm{In}_{0.15} \mathrm{Ga}_{0.85} \mathrm{~N} \mathrm{QW}$ and $12-\mathrm{nm} \mathrm{GaN}$ barriers $\left(T_{g}=740^{\circ} \mathrm{C}\right)$. The growth of the active regions and barriers employed $\mathrm{N}_{2}$ as the carrier gas with a flow rate of $2500 \mathrm{sccm}$. The molar flow rates of triethylgallium (TEGa) and trimethylindium (TMIn) were 1.104 and $0.613 \mu \mathrm{mol} / \mathrm{min}$, respectively. The In-content of the InGaN QW studied here was found to be $15 \%$, as calibrated via X-ray diffraction.

\section{LED DEVICE CHARACTERISTICS}

Fig. 9 shows the LED light output power versus injection current of three comparison LEDs. The LED devices were measured under continuous wave $(\mathrm{CW})$ conditions at room 


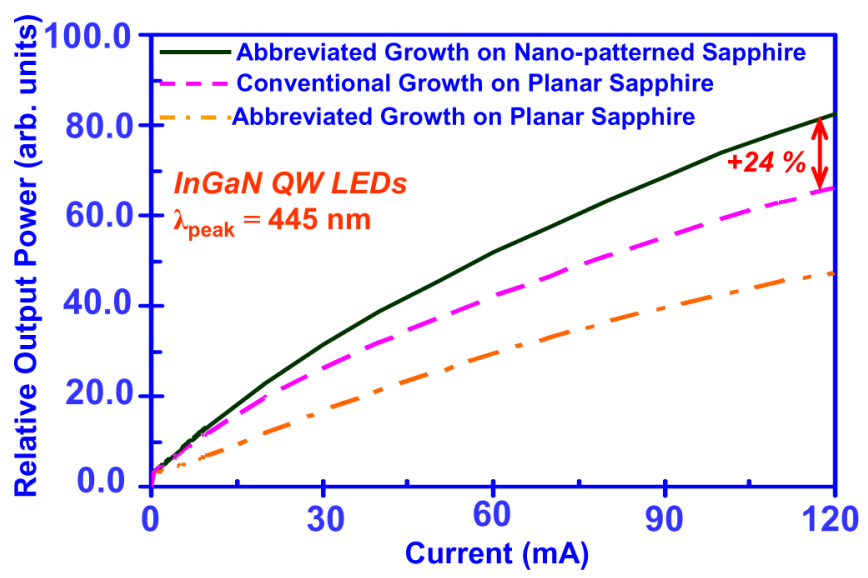

Fig. 9. Comparison of room temperature $\mathrm{CW}$ output power-injection current of $\mathrm{In}_{0.15} \mathrm{Ga}_{0.85} \mathrm{~N}$ QW LEDs grown on three comparison templates (samples 1, 2, and 3).

temperature. The on-wafer output power of the LEDs with an area of $1.25 \times 10^{-3} \mathrm{~cm}^{2}$ was measured in a dark room. Not surprisingly, the InGaN QWs LED grown using abbreviated growth mode on planar sapphire (sample 3 ) had an output power $28 \%$ lower than the LED grown using the conventional method on planar sapphire (sample 2). However, for the InGaN QWs LED grown using abbreviated mode on patterned AGOG sapphire (sample 1), the output power and internal quantum efficiency were enhanced by $24 \%$ in comparison to the conventional LED (sample 2).

Fig. 10(a) and (b) show cross-sectional transmission electron microscopy (TEM) images for LED device structures on GaN template grown on planar sapphire by using conventional method (sample 2) and LED device structures on GaN template grown on nanopatterned AGOG sapphire by using abbreviated growth mode (sample 1), respectively. These preliminary measurements indicate that the threading dislocation density of the GaN grown on patterned AGOG sapphire is approximately an order magnitude lower than that of $\mathrm{GaN}$ grown on planar sapphire. The reduction in dislocation density can be observed in Fig. 10(b); however, it is difficult to provide accurate quantification of the threading dislocation density from cross-sectional TEM. Future works on plan-view [0001] TEM measurements [19] on both sample 1 and sample 2 are required to provide accurate quantification of the dislocation density.

This proof-of-concept experiment demonstrates that the abbreviated growth mode on patterned sapphire formed via the AGOG process shows promise for achieving improved radiative efficiency of InGaN QW LEDs. At this stage, it must be acknowledged that electron beam patterning of the substrate is impractical as a production tool. The advantage offered by the use of patterned AGOG sapphire substrates can be exploited only when the patterning can be accomplished by large-scale and low-cost lithography processes (e.g., holography lithography). Once this is achieved, the thermal processing of the substrates can be performed as a batch process at relatively low additional cost.

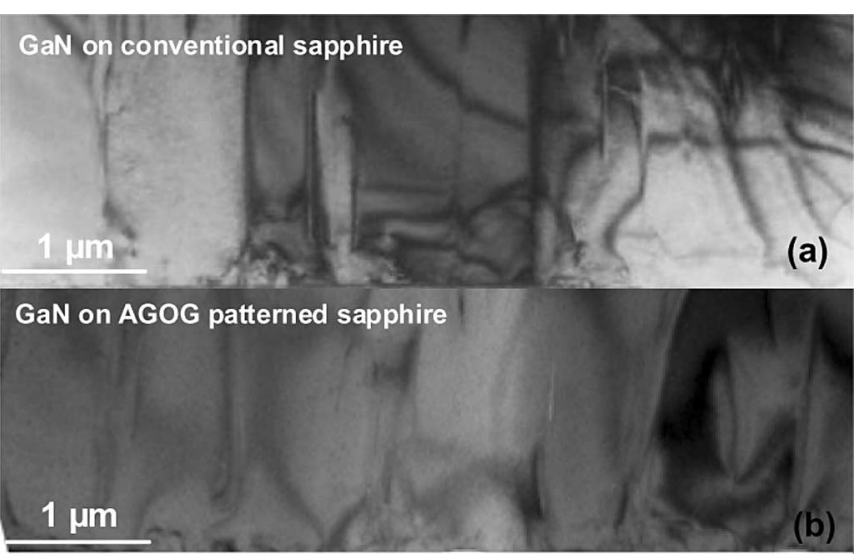

Fig. 10. Cross-sectional transmission electron microscopy of (a) GaN grown on planar sapphire using conventional growth and (b) GaN grown on nanopatterned sapphire with abbreviated growth mode.

\section{CONCLUSION}

In summary, proof-of-concept MOVPE growth experiments were conducted with GaN template on nanopatterned AGOG sapphire substrates. The use of patterned AGOG sapphire enabled introduction of an "abbreviated growth mode" that significantly reduces the epitaxy time for a typical GaN template. This growth mode is dependent on the patterned sapphire surface for success. Although a thin-GaN buffer layer is still needed to facilitate coalescence of the high-temperature GaN, the conventional etch-back and recovery steps are no longer required.

Comparison studies were carried out on various InGaN QW LED devices grown on three comparison GaN templates: abbreviated growth mode on nanopatterned AGOG sapphire, conventional mode on planar sapphire, and abbreviated growth mode on planar sapphire. The output power of the LEDs grown with the abbreviated mode on the nanopatterned substrate demonstrated improved optical output power of $24 \%$ over the LEDs grown using the conventional method. This improvement can be attributed to the increase in radiative efficiency of the material. Further structural characterizations are still required to provide a better understanding of the mechanism underlying this improvement. In addition to the improved radiative efficiency in InGaN/GaN LED devices, the abbreviated growth mode of $\mathrm{GaN}$ template on patterned AGOG sapphire-accompanied by largescale and low-cost substrate patterning-could potentially lead to a significant reduction in the growth time and cost for LED epitaxy.

\section{REFERENCES}

[1] S. Nakamura and T. Mukai, "High-quality InGaN films grown on GaN films," Jpn. J. Appl. Phys., vol. 31, pp. L1457-L1459, 1992.

[2] H. Amano, N. Sawaki, I. Akasaki, and Y. Toyoda, "Metalorganic vapor phase epitaxial growth of a high quality GaN film using an AlN buffer layer," Appl. Phys. Lett., vol. 48, pp. 353-355, 1986.

[3] I. Akasaki, "The evolution of group III nitride semiconductors: Seeking blue light emission," Mat. Sci. Eng. B, vol. 74, pp. 101-106, 2000.

[4] D. D. Koleske, A. J. Fischer, A. A. Allerman, C. C. Mitchell, K. C. Cross, S. R. Kurtz, J. J. Figiel, K. W. Fullmer, and W. G. Breiland, "Improved brightness of $380 \mathrm{~nm} \mathrm{GaN}$ light emitting diodes through intentional delay of the nucleation island coalescence," Appl. Phys. Lett., vol. 81, pp. 1940$1942,2002$. 
[5] S. Nakamura, "The roles of structural imperfections in InGaN-based blue light-emitting diodes and laser diodes," Science, vol. 281, pp. 956-961, 1998.

[6] S. Nakamura, M. Senoh, S. Nagahama, N. Iwasa, T. Yamada, T. Matsushita, H. Kiyoku, Y. Sugimoto, T. Kozaki, H. Umemoto, M. Sano, and K. Chocho, "InGaN/GaN/AlGaN-based laser diodes with modulationdoped strained-layer superlattices grown on an epitaxially laterally overgrown GaN substrate,” Appl. Phys. Lett., vol. 72, pp. 211-213, 1998.

[7] P. Kozodoy, J. P. Ibbetson, H. Marchand, P. T. Fini, S. Keller, J. S. Speck, S. P. DenBaars, and U. K. Mishra, "Electrical characterization of GaN p-n junctions with and without threading dislocations," Appl. Phys. Lett., vol. 73, pp. 975-977, 1998.

[8] M. Hansen, P. Fini, M. Craven, B. Heying, J. S. Speck, and S. P. DenBaars, "Morphological and optical properties of InGaN laser diodes on laterally overgrown GaN," J. Cryst. Growth, vol. 234, pp. 623-630, 2002.

[9] A. M. Roskowski, E. A. Preble, S. Einfeldt, P. M. Miraglia, and R. F. Davis, "Investigations regarding the maskless pendeo-epitaxial growth of $\mathrm{GaN}$ films prior to coalescence," IEEE J. Quantum Electron., vol. 38, no. 8, pp. 1006-1016, Aug. 2002.

[10] C. I. H. Ashby, C. C. Mitchell, J. Han, N. A. Missert, P. P. Provencio, D. M. Follstaedt, G. M. Peake, and L. Griego, "Low-dislocation-density GaN from a single growth on a textured substrate," Appl. Phys. Lett., vol. 77, pp. 3233-3235, 2000 .

[11] D. Zubia, S. H. Zaidi, S. R. J. Brueck, and S. D. Hersee, "Nanoheteroepitaxial growth of GaN on Si by organometallic vapor phase epitaxy," Appl. Phys. Lett., vol. 76, pp. 858-860, 2000.

[12] T. S. Kim, S. M. Kim, Y. H. Jang, and G. Y. Jung, "Increase of light extraction from $\mathrm{GaN}$ based light emitting diodes incorporating patterned structure by colloidal lithography," Appl. Phys. Lett., vol. 91, Art no. 171114, 2007

[13] D. H. Kang, J. C. Song, B. Y. Shim, E. A. Ko, D. W. Kim, S. Kannappan, and C. R. Lee, "Characteristic comparison of GaN grown on patterned sapphire substrates following growth time," Jap. J. App. Phys., vol. 46, pp. 2563-2566, 2007.

[14] H. Kudo, Y. Ohuchi, T. Jyouichi, T. Tsunekawa, H. Okagawa, K. Tadatomo, Y. Sudo, M. Kato, and T. Taguchi, "Demonstration of high-efficient InGaN-based violet light-emitting diodes with an externalquantum efficiency of more than 40\%," Phys. Stat. Sol. (a), vol. 200, pp. 95-98, 2003.

[15] J. Lee, D. H. Kim, J. Kim, and H. Jeon, "GaN-based light-emitting diodes directly grown on sapphire substrate with holographically generated twodimensional photonic crystal patterns," Curr. Appl. Phys., vol. 9, pp. 633$635,2009$.

[16] H. Park, H. M. Chan, and R. P. Vinci, "Patterning of sapphire substrates via a solid state conversion process," J. Mater. Res., vol. 20, pp. 417-423, 2004.

[17] Y. K. Ee, R. A. Arif, N. Tansu, H. Li, H. M. Chan, R. P. Vinci, P. Capek, N. Jha, and V. Dierolf, "Improved photoluminescence of InGaN quantum wells grown on nano-patterned AGOG sapphire substrate by metalorganic vapor phase epitaxy," in Proc. IEEE Lasers Electro-Opt. Soc. (LEOS'07), vol. 21, pp. 902-903.

[18] Z. H. Feng and K. M. Lau, "Enhanced luminescence from GaN-based blue LEDs grown on grooved sapphire substrates," IEEE Photon. Technol. Lett., vol. 17, no. 9, pp. 1812-1814, Sep. 2005

[19] D. M. Follstaedt, N. A. Missert, D. D. Koleske, C. C. Mitchell, and K. C. Cross, "Plan-view image contrast of dislocations in GaN," Appl. Phys. Lett., vol. 83, pp. 4797-4799, 2003.

Yik-Khoon Ee (S'08) received the Bachelor's degree (with first-class honors) in electrical and electronic engineering from Nanyang Technological University, Singapore, in 2003, and the M.S. degree from Lehigh University, Bethlehem, $\mathrm{PA}$, in 2007. He is currently working toward the Ph.D. degree at Lehigh University.

In 2003, he was with Denselight Semiconductors and Agilent Technologies. Since June 2005, he has been a Graduate Student in the Department of Electrical and Computer Engineering, Lehigh University. His current research interests include field of semiconductor optoelectronics, metal-organic chemical vapor deposition (MOCVD) epitaxy, device fabrication of III-Nitride LEDs, dislocation density reduction of III-Nitride material on sapphire, and using novel techniques to increase the light extraction efficiency of LEDs. He is the author and coauthor of more than 46 papers published in refereed journal and conference publications.

Mr. Ee was a recipient of the Best Student Paper Award on NanoPhotonics at IEEE Photonics Global Conference 2008.
Jeffrey M. Biser received the B.S. degree in materials science and engineering, concentrating on Polymers, from Cornell University, Ithaca, NY, in 2003. He is currently working toward the Ph.D. degree at the Materials Science Department, Lehigh University, Bethlehem, PA.

He is with the Materials Science Department, Lehigh University, where he studied various topics related to the morphological changes at elevated temperatures of aluminum films and nanostructures on sapphire surfaces. His work has appeared in the Journal of Applied Physics.

Mr. Biser has presented his work at American Ceramics Society Conferences at International Conferences on Modern Materials and Technologies (CIMTEC), Acireale, Italy, 2006.

Wanjun Cao received the B.S. degree in materials science and engineering from Zhejiang University, Hangzhou, China, in 2007.

Since 2007, he has been a Graduate Student in the Materials Science Department, Lehigh University, Bethlehem, PA.

His current research interests include the field of nanostructures on sapphire and silicon substrates and semiconductor thin film growth.

Helen M. Chan received the B.Sc. (with first-class honors) and Ph.D. degrees from the Department of Materials Science and Technology, Imperial College, University of London, London, U.K.

In 1986, she joined Lehigh University, Bethlehem, PA, where she is currently the New Jersey Zinc Chair and the Chairperson of the Department of Materials Science and Engineering. She took an 18-month leave of absence from Lehigh University and worked with the National Institute of Standards and Technology, where she was engaged in the Mechanical Properties Group of the Ceramics Division. She returned to Lehigh University in January 1988, and was promoted to the rank of Associate Professor with tenure in 1991 and to a Full Professor in 1995. Her current research interests include the application of reactive processing to fabricate unique ceramic/metal structures, including cellular and nanopatterned materials. She is also actively involved in research on the role of dopants and interfacial chemistry on diffusion limited processes in ceramics. She is the author or coauthor of more than 165 publications. She is also an Editor of the Journal of Materials Science, and has been an Associate Editor for the Journal of the American Ceramic Society since 1999.

Prof. Chan has received the American Ceramic Society Roland B. Snow award on five separate occasions (1986, 1990, 1992, 1999, and 2000). In 1990, she was awarded the Alfred Noble Robinson Award for "outstanding performance and unusual promise of professional achievement," and she has received Lehigh University's "Service Teaching Excellence Award" on three separate occasions (1991, 1992, and 2007). She was named the 1992 recipient of the American Society for Metals (ASM) International's Bradley Stoughton Award for outstanding young faculty in the field of materials science and engineering. In 1993, she was awarded the "Class of 1961" Professorship by Lehigh University for "distinction in teaching, research and service." She was inducted as a Fellow of the American Ceramic Society (2005), and received Lehigh University's 2005 Eleanor and Joseph F. Libsch Award for excellence in research. Most recently, She was the Chair of the 2008 Gordon Research Conference on Solid State Ceramics. She is included in Thomson ISI's list of highly cited researchers in materials. 
Richard P. Vinci received the undergraduate degree in materials science and engineering from Massachusetts Institute of Technology (MIT), Cambridge, in 1988, and the Ph.D. degree from the Department of Materials Science and Engineering, Stanford University, Palo Alto, CA, in 1994.

He was an Acting Assistant Professor in the Department of Materials Science and Engineering, Stanford University. In 1998, he joined Lehigh University, Bethlehem, PA, where he was a P.C. Rossin Assistant Professor from 2001 to 2003 and the Class of 1961 Associate Professor of materials science and engineering from 2004 to 2006, and is currently an Associate Professor and the Director of the Nano- and Micro-Mechanical Behavior Laboratory, Center for Advanced Materials and Nanotechnology, Department of Materials Science and Engineering. His current research interests include the processing and properties of thin films and small-scale structures, with an emphasis on mechanical behavior, fabrication and application of microelectromechanical systems (MEMS) devices for mechanical measurements, strengthening mechanisms in platinum, gold, and aluminum thin films, viscoelasticity and stress relaxation mechanisms in nanoscale metal films, cryogenic behavior of thin films, and nanopatterning of metal films for solid state conversion to form single-crystal oxides. He also explores the mechanical behavior of small volumes of natural materials, including human tissue.

Dr. Vinci has received the American Society for Metals (ASM) International's Bradley Stoughton Award for Young Teachers, the Outstanding Young Member Award from the Lehigh Valley Chapter of ASM, the Lehigh University Junior Award for Distinguished Teaching, the P.C. Rossin College of Engineering Teaching Excellence Award, and his department's Gilbert E. Doan Award given by the graduating senior class. He has organized numerous professional symposia, including the 2008 Gordon Research Conference on Thin Film and Small-Scale Mechanical Behavior.
Nelson Tansu (S'99-M'02) was born on October 1977. He received the B.S. degree (with highest distinction) in applied mathematics, electrical engineering, and physics, and the Ph.D. degree in electrical engineering from the University of Wisconsin-Madison, Madison, in May 1998 and May 2003, respectively.

From July 2003 till April 2009, he was an Assistant Professor and Peter C. Rossin Assistant Professor (Term Chair 2007-2009) in the Department of Electrical and Computer Engineering (ECE) and the Center for Optical Technologies (COT) at Lehigh University. Beginning May 2009 (till present), he has been appointed as Associate Professor (with Tenure) of electrical and computer engineering at Lehigh University. He is engaged in research on the theoretical and experimental aspects of the physics of semiconductor optoelectronics materials and devices, the physics of low-dimensional semiconductor (nanostructure), and metal-organic chemical vapor deposition (MOCVD), and device fabrications of III-nitride and III-V-nitride semiconductor optoelectronics devices on GaAs, InP, and $\mathrm{GaN}$ substrates. He has authored or coauthored numerous refereed international journal and conference publications (total $>160$ ), and he also currently holds several U.S. patents.

Dr. Tansu was a panel member for the U.S. National Science Foundation, the U.S. Department of Defense, and other agencies in U.S. and abroad. He has also given numerous lectures, seminars, and invited talks (total $>35$ ) in universities, research institutions, and conferences in USA, Canada, Europe, and Asia. He is the Primary Guest Editor of the IEEE JouRnal OF SELECTED ToPICS IN QUANTUM Electronics Special Issue on Solid State Lighting in 2008-2009, and he also serves as an Associate Editor for IEEE PHOTONICS JouRNAL (2009-present) and as Assistant/Associate Editor for Nanoscale Research Letters (2007-present). Dr. Tansu was also an invited General Participant at the 2008 National Academy of Engineering (NAE)'s U.S. Frontiers of Engineering (FOE) Symposium, and he also serves as the Organizing Committee for the 2009 NAE's U.S. Frontiers of Engineering Symposium. He was a recipient of the Bohn Scholarship, the WARF Graduate University Fellowship, the Vilas Graduate University Fellowship, and the Graduate Dissertator Travel Funding Award, the 2003 Harold A. Peterson ECE Best Research Award (1st Prize) at the University of Wisconsin-Madison, and the 2008 Libsch Early Career Research Award at Lehigh University. 